\title{
The Long-term Outcome of Sleeve Gastrectomy in Managing Obstructive Sleep Apnea in Prader-Willi Syndrome Patients: A Case Series
}

\author{
Safa Eltahir ${ }^{*},{ }^{(\mathbb{D}}$, Mohammed Alzaid $^{\mathbb{D}}$, Gawahir Mukhtar, Wadha Alotaibi ${ }^{(\mathbb{D}}$ \\ Pediatric Pulmonology Section, Children Specialised Hospital, King Fahad Medical City, Riyadh, Saudi Arabia
}

\section{ARTICLE INFO}

\section{Article History}

Received 23 June 2020

Accepted 28 August 2020

\section{Keywords}

Gastric sleeve surgery

OSA

PWS

\begin{abstract}
Background: Prader-Willi syndrome (PWS) is a genetic disorder with multisystem involvement. Bariatric laparoscopic Sleeve Gastrectomy (SG) surgery has been proposed as a means of weight control in PWS patients. There is some controversy around sleeve gastrectomy as a management strategy in PWS to control Obstructive Sleep Apnea (OSA).

Case-Series: In this case series we are questioning the effectiveness of bariatric gastric sleeve surgery as a long-term solution for weight control. Therefore, we followed up four patients with PWS in our center who had SG and evaluated their growth parameters along with symptoms of sleep disordered breathing. The median age of patients in this group was 7.5 years. There were three males and one female. The average Body Mass Index (BMI) prior to SG was 40.5. The average BMI after SG, at 5-7 years, was 40.8. This shows an insignificant effect of SG on BMI and control of OSA on follow-up over more than 5 years.

Conclusion: We believe that SG has limited and transient success for obesity management in PWS patients, especially in the absence of a multidisciplinary team that can provide behavioral therapy, nutritional plan, and psychosocial support. Further studies are necessary to compare the long-term outcome sleeve gastrectomy with behavioral and strict nutritional therapy.
\end{abstract}

(C) 2020 Dr. Sulaiman Al Habib Medical Group. Publishing services by Atlantis Press International B.V. This is an open access article distributed under the CC BY-NC 4.0 license (http://creativecommons.org/licenses/by-nc/4.0/).

\section{INTRODUCTION}

Prader-Willi syndrome (PWS) is a genetic disorder with multisystem involvement that is a consequence of a defect in the expression of paternal copy of chromosome 15 [1]. Those affected progress from neonatal hypotonia with feeding difficulty to morbid obesity caused by hyperphagia. Hyperphagia is one of the hallmarks of the disease, and it is attributed to hypothalamic dysfunction leading to lack of satiety [1-3]. Cardiopulmonary complications of obesity are the most common cause of morbidity and mortality in patients with PWS [4]. They develop alveolar hypoventilation related to respiratory muscle weakness and upper airway obstruction attributed to fat deposition in the pharyngeal wall and upper airway dilator muscles weakness. This constellation of findings eventually leads to Obstructive Sleep Apnea (OSA) and Pulmonary Hypertension (PHT) $[5,6]$.

Part of the management of such patients is controlling their weight. Bariatric laparoscopic Sleeve Gastrectomy (SG) surgery has been proposed as a means of weight control [7-9]. In this study, we are questioning the effectiveness of bariatric gastric sleeve surgery as a long-term solution for weight control and prevention of subsequent cardiopulmonary complications in a series of four cases.

Corresponding author. Email: seltahir@kfmc.med.sa

Peer review under responsibility of the Dr. Sulaiman Al Habib Medical Group

\section{CASE 1}

An 8-year-old boy, known to have PWS, which was confirmed by methylation test, presented to our pediatric sleep clinic with morbid obesity and OSA. His body weight was $43 \mathrm{~kg}$, height $107 \mathrm{~cm}$, and Body Mass Index (BMI) 37.5. He was diagnosed clinically with severe OSA using the pediatric sleep questionnaire because Polysomnography (PSG) was not available at that time. His blood gas showed $\mathrm{CO}_{2}$ retention, overnight pulse oximetry revealed severe hypoxemia, and echocardiography showed evidence of mild PHT with a pressure gradient of $35 \mathrm{mmHg}$. An Adenotonsillectomy (AT) led to a temporary improvement. However, over the following year his OSA symptoms worsened because he continued to gain weight (up to $53 \mathrm{~kg}$, height $110 \mathrm{~cm}$, BMI 43.8), and he became wheelchair-bound despite receiving multidisciplinary management to control his weight. The family failed to maintain food restriction at home and school. At this point, he underwent bariatric gastric sleeve surgery. Postoperatively, the patient lost $16.4 \mathrm{~kg}$, which was accompanied by remission of his OSA symptoms within 8 months. Follow-up echocardiography showed no evidence of Pulmonary Hypertension (PHTN) with a pressure gradient of $25 \mathrm{mmHg}$. Unfortunately, long-term follow-up after SG showed a steady increase in his body weight (Figure 1) and BMI (Figure 2), especially when commenced on growth hormone therapy for short stature. Eventually, he started having symptoms of OSA, which was confirmed by a diagnostic PSG that revealed an Apnea-Hypopnea Index (AHI) of 7, and 


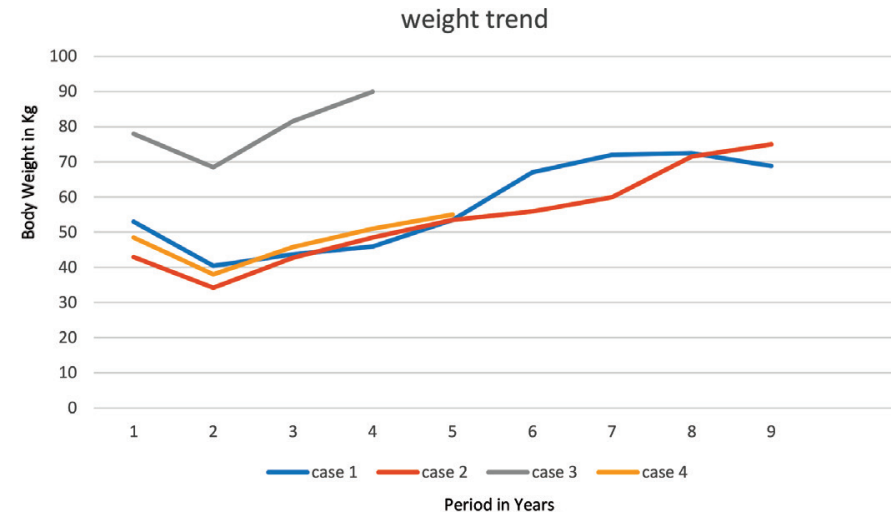

Figure 1 Trend of patient's weight.

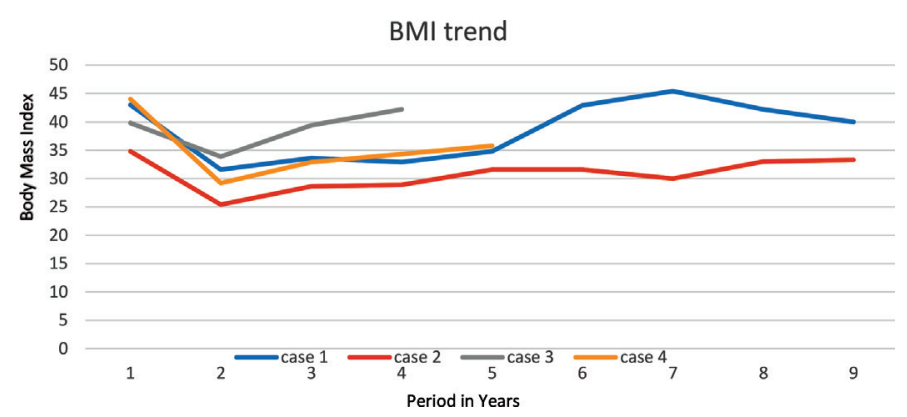

Figure 2 Trend of patient's body mass index.

he needed a Continuous Positive Airway Pressure (CPAP) with a Positive End-Expiratory Pressure (PEEP) of $6 \mathrm{cmH}_{2} \mathrm{O}$ based on his titration study. A follow-up titration study conducted 4 years later showed that he needed a higher PEEP $\left(9 \mathrm{cmH}_{2} \mathrm{O}\right)$.

\section{CASE 2}

A 4-year-old boy, known to have PWS, confirmed by methylation test, was referred initially to our clinic. His symptoms were suggestive of OSA that was confirmed by pediatric sleep questionnaire as no PSG was available at the time. It was attributed to the patient's obesity; his body weight was $28.5 \mathrm{~kg}$, height $104 \mathrm{~cm}$, BMI 26.3. Over the next year, as he had a steady increase in weight, his OSA symptoms progressively worsened. Early morning blood gases showed $\mathrm{CO}_{2}$ retention, overnight pulse oximetry showed significant hypoxemia, echocardiography showed severe PHTN with supra systemic pressure gradient of more than $120 \mathrm{mmHg}$, so the patient was started on sildenafil. Later on, he presented with severe pneumonia and cardiac arrest. He was mechanically ventilated initially and eventually weaned to Bilevel Positive Airway Pressure (BiPAP) ventilation. After the condition stabilized, he underwent SG together with nasal septostomy and partial inferior turbinectomy with no complications. In the subsequent 2 months, he lost $13 \mathrm{~kg}$ and his weight remained between 34 and $36 \mathrm{~kg}$ over the next 18 months after SG (Figure 1). The follow-up echocardiography showed no evidence of PHTN, and sildenafil stopped 1 year after SG. Moreover, there was no evidence of hypoxemia on overnight pulse oximetry, and serial blood gases were within normal limits. Eighteen months after SG, he had progressive weight gain (Figure 1) and manifested OSA symptoms again. A follow-up echocardiography showed trivial tricuspid regurgitation and mild right ventricular dilatation. A PSG study showed severe OSA with an AHI of 55.1, and a follow-up titration study showed that he needed CPAP with a PEEP of $8 \mathrm{cmH}_{2} \mathrm{O}$ (Table 1).

\section{CASE 3}

A 9-year-old male child, who was diagnosed with PWS according to consensus diagnostic criteria, had morbid obesity (body weight $78 \mathrm{~kg}$, height $142 \mathrm{~cm}$, BMI 38.7) and severe OSA on oxygen therapy during sleep, complicated by PHTN. He was admitted through the emergency department with congested heart failure and pulmonary edema, which required BiPAP. He was started on captopril and furosemide for his PHTN. His blood gas showed type 2 respiratory failure ( $\mathrm{pH} 7.29, p \mathrm{CO}_{2} 81.3, \mathrm{HCO}_{3} 38 \mathrm{mmol} / \mathrm{L}$ ), and investigations revealed dyslipidemia and prediabetes. Echocardiography showed severe PHTN with pressure gradient of $53 \mathrm{mmHg}$ and estimated right ventricular of $58 \mathrm{mmHg}$, which was almost equal to $50 \%$ of systemic pressure. PSG with BiPAP titration study showed severe OSA, which required $19 / 13 \mathrm{cmH}_{2} \mathrm{O}$ pressure, with $2 \mathrm{~L} /$ min oxygen to maintain oxygen saturation $>94 \%$ during sleep time. Later on, when the patient's condition stabilized, he underwent an uneventful SG. He lost almost $10 \mathrm{~kg}$ and achieved nadir weight of $68.45 \mathrm{~kg}$ over the next 9 months with complete resolution of dyslipidemia and prediabetes status. He ceased oxygen therapy at 4 months after SG, and then BiPAP at 6 months. However, upon follow-up, he had progressive weight gain (Figure 1). Despite psychosocial therapy, the family failed to keep him on a restricted weight-reducing program. Eventually, nocturnal BiPAP resumed again, when the patient manifested severe OSA that was confirmed by PSG, with AHI of 13.6 (Table 1), which required adjusting BiPAP to 24/13 during sleep time with $2 \mathrm{~L} / \mathrm{min}$ oxygen at 4 years after SG.

\section{CASE 4}

A 5-year-old female child, who has PWS according to consensus diagnostic criteria for PWS, presented to our emergency department with severe respiratory distress and cyanosis. She had morbid obesity, body weight $38.6 \mathrm{~kg}$, height $108 \mathrm{~cm}$, and BMI 33.1. She also complained of severe OSA diagnosed by PSG. Initial capillary blood gases (CBG) showed $\mathrm{CO}_{2}$ retention $\left(\mathrm{pH} 7.333, \mathrm{COO}_{2} 56 \mathrm{mmHg}, \mathrm{HCO}_{3}\right.$ $29.2 \mathrm{mmol} / \mathrm{L}$ ). Overnight pulse oximetry showed severe hypoxemia. She was started on CPAP of $6 \mathrm{cmH}_{2} \mathrm{O}$ pressure. Echocardiography showed mild PHTN with a pressure gradient of $33 \mathrm{mmHg}$. Upon follow-up over the next 6 months, she was steadily gaining weight up to $48.5 \mathrm{~kg}$ (Figure 1), height $105.8 \mathrm{~cm}$, and BMI 43.32 (Figure 2); despite her multidisciplinary management, the family failed to sustain a strict food regimen, which resulted in worsening of her OSA and PHTN. On a follow-up, her echocardiography showed right atrial dilatation and the tricuspid regurgitation gradient pressure was $45 \mathrm{mmHg}$, so she was started on sildenafil. After her condition stabilized, she underwent SG without complications. After SG, the patient's weight gradually decreased to a nadir weight of $36.6 \mathrm{~kg}$ (Figure 1), height $112 \mathrm{~cm}$, BMI 29.17 (Figure 2) by 5 months, which was associated with remission of OSA and cessation of her CPAP therapy. However, she had a steady weight increment over the next 
Table 1 Post-SG trend of BMI, OSA severity, and outcomes

\begin{tabular}{|c|c|c|c|c|c|c|c|c|c|}
\hline \multirow[b]{2}{*}{ Cases } & \multicolumn{2}{|c|}{ At SG } & \multicolumn{7}{|c|}{ Follow-up after SG } \\
\hline & Age (years) & BMI & BMI & $\begin{array}{c}\text { Age at PSG } \\
\text { (years) }\end{array}$ & AHI & Type of NIV & $\begin{array}{l}\text { Pressure } \\
\left(\mathrm{cmH}_{2} \mathrm{O}\right)\end{array}$ & $\begin{array}{c}\text { Age at ECHO } \\
\text { (years) }\end{array}$ & $\begin{array}{l}\text { Echo } \\
\text { findings }\end{array}$ \\
\hline 1 & 9 & 43.8 & $\begin{array}{l}\text { Year } 1,31.63 \\
\text { Year 3, 32.96 } \\
\text { Year 5, 42.88 } \\
\text { Year 7, 45.35 }\end{array}$ & 13 & 7.0 & CPAP & 9 & NA & $\begin{array}{l}\text { No clinical } \\
\text { indication to } \\
\text { be repeated }\end{array}$ \\
\hline 2 & 6 & 34.6 & $\begin{array}{l}\text { Year } 1,25.41 \\
\text { Year 3, 28.92 } \\
\text { Year 5, 31.60 } \\
\text { Year 7, 32.77 }\end{array}$ & 12 & 55.1 & CPAP & 8 & NA & $\begin{array}{l}\text { Lost to } \\
\text { follow-up }\end{array}$ \\
\hline 3 & 9.5 & 39.7 & $\begin{array}{c}\text { Year } 1,-33.94 \\
\text { Year } 3,39.62 \\
\text { Year 5, } 44.31\end{array}$ & 14 & 13.6 & BiPAP & $24 / 13$ & 13 & $\begin{array}{l}\text { Pulmonary } \\
\text { hypertension }\end{array}$ \\
\hline 4 & 5.7 & 44 & $\begin{array}{l}\text { Year 1, } 29.23 \\
\text { Year 3, } 34.26 \\
\text { Year 5, } 40.92\end{array}$ & 9 & 50.9 & CPAP & 9 & 10 & $\begin{array}{l}\text { Pulmonary } \\
\text { hypertension }\end{array}$ \\
\hline
\end{tabular}

NIV, noninvasive ventilation

year (Figure 1) with the occurrence of her OSA symptoms. At 3 years after SG, she had regained almost all of her weight before the SG procedure $(48 \mathrm{~kg})$ and developed severe OSA, which was confirmed by PSG (AHI 50.9) (Table 1). She needed to resume CPAP with a pressure of $9 \mathrm{mmHg}$ during sleep according to her titration study.

\section{DISCUSSION}

Prader-Willi syndrome is one of the most common syndromic obesities $[1,2]$. Although obesity is multifactorial, the main cause is compulsive hyperphagia, which is attributed to hypothalamic dysfunction leading to a lack of satiety [1-3]. Patients develop aggressive food-seeking behavior, manifesting in hoarding, stealing food, and lying about it. They also might gobble their meals, which can lead to choking and aspiration $[3,10,11]$.

Sleep-related Disorder of Breathing (SRDB)-related cardiopulmonary complications are the most common cause of morbidity and mortality in patients with PWS [4]. SRDB is common in patients with PWS, with a prevalence of $79.9 \%$ [6]. It is attributed to OSA that is caused by fat deposition in the pharyngeal wall, abnormal craniofacial characteristics, and adenotonsillar hypertrophy. Other causes of SRDB include central apneas and hypoventilation syndrome caused by generalized hypotonia $[6,12]$. In our cohort, all patients had OSA complicated with PHT in varying severity. Two patients further progressed into congestive heart failure and cardiac arrest. So, a decision was made to proceed with laparoscopic gastric sleeve surgery to manage their morbid obesity, which had good results in the form of weight loss, improving symptoms of SRDB and its related complications, particularly in the first year after gastric sleeve surgery.

Unfortunately, these results were temporary, as our patients started to regain weight (Figures 1 and 2). We believe this was because obesity in PWS patients is centrally derived and without strict dietary control and behavioral therapy, they will not be able to keep their weight under control. It is worth mentioning that all cases were being followed up with a psychologist and a dietician. However, parents were unable to implement the dietary restrictions which are attributable to the difficulties in dealing with PWS patients and the parents being older and having a lower educational level. All patients eventually exhibited symptoms of OSA, which was later confirmed by a PSG that mandated an intervention by noninvasive ventilation at home. Two patients were further complicated by PHT (Table 1).

There are conflicting reports about the effectiveness of bariatric sleeve surgery in managing the weight of patients with PWS. Alqahtani et al. [9] reported significant weight reduction and control of comorbidities with no significant complications. Other studies report that surgical interventions have a short-term success and that this does not justify subjecting PWS patients to surgical interventions and their potential complications [8]. Furthermore, weight control is better achieved with strict dietary restriction of caloric intake with macronutrient supplementation, behavioral therapy, and a daily exercise regimen $[8,9]$.

In conclusion, we are questioning the long-term outcome of bariatric gastric sleeve surgery in the management of obesity and its complications in PWS patients. We believe that it has a transient effect, especially in the absence of a multidisciplinary team to provide behavioral therapy, nutritional plan, and psychosocial support. Further studies are warranted to compare the long-term outcome of behavioral therapy and gastric sleeve surgery.

\section{CONFLICTS OF INTEREST}

The authors declare they have no conflicts of interest.

\section{AUTHORS' CONTRIBUTION}

SE and WA contributed in study conception and design. SE, MA, GM and WA contributed in acquisition of data. SE, MA and WA contributed in analysis and interpretation of data. SE, MA and GM contributed in drafting the manuscript. Critical revision was carried out by SE, MA, GM and WA. 


\section{REFERENCES}

[1] Cassidy SB, Schwartz S, Miller JL, Driscoll DJ. Prader-Willi syndrome. Genet Med 2012;14;10-26.

[2] Butler MG, Theodoro MF, Bittel DC, Donnelly JE. Energy expenditure and physical activity in Prader-Willi syndrome: comparison with obese subjects. Am J Med Genet A 2006; 143A;449-59.

[3] Elena G, Bruna C, Benedetta M, Stefania DC, Giuseppe C. PraderWilli syndrome: clinical aspects. J Obes 2012;2012;473941.

[4] Butler MG, Manzardo AM, Heinemann J, Loker C, Loker J. Causes of death in Prader-Willi syndrome: Prader-Willi Syndrome Association (USA) 40-year mortality survey. Genet Med 2017;19;635-42.

[5] Eiholzer U. Deaths in children with Prader-Willi syndrome. A contribution to the debate about the safety of growth hormone treatment in children with PWS. Horm Res 2005;63;33-9.

[6] Sedky K, Bennett DS, Pumariega A. Prader Willi syndrome and obstructive sleep apnea: co-occurrence in the pediatric population. J Clin Sleep Med 2014;10;403-9.
[7] Kobayashi J, Kodama M, Yamazaki K, Morikawa O, Murano S, Kawamata N, et al. Gastric bypass in a Japanese man with PraderWilli syndrome and morbid obesity. Obes Surg 2003;13;803-5.

[8] Scheimann AO, Butler MG, Gourash L, Cuffari C, Klish W. Critical analysis of bariatric procedures in Prader-Willi syndrome. J Pediatr Gastroenterol Nutr 2008;46;80-3.

[9] Alqahtani AR, Elahmedi MO, Al Qahtani AR, Lee J, Butler MG. Laparoscopic sleeve gastrectomy in children and adolescents with Prader-Willi syndrome: a matched-control study. Surg Obes Relat Dis 2016;12;100-10.

[10] Butler MG. Prader-Willi syndrome: obesity due to genomic imprinting. Curr Genomics 2011;12;204-15.

[11] Khan MJ, Gerasimidis K, Edwards CA, Shaikh MG. Mechanisms of obesity in Prader-Willi syndrome. Pediatr Obes 2018;13; 3-13.

[12] Al-Saleh S, Al-Naimi A, Hamilton J, Zweerink A, Iaboni A, Narang I. Longitudinal evaluation of sleep-disordered breathing in children with Prader-Willi Syndrome during 2 years of growth hormone therapy. J Pediatr 2013;162;263.e1-8.e1. 